acta medica

Gamze Durhan, MD

ORCID: 0000-0002-6281-9287

nti-neutrophil cytoplasmic antibody (ANCA) associated vasculitides are multisystem disorders characterized by necrotizing vasculitis which predominantly affects small vessels. The three major clinicopathological variants of ANCA-associated vasculitides are granulomatosis with polyangiitis (GPA), eosinophilic granulomatosis with polyangiitis (EGPA) and microscopic polyangiitis (MPA). While EGPA and GPA present as necrotizing granulomatous inflammation, MPA presents as nongranulomatous necrotizing inflammation $[1,2]$.

Although combined manifestations are more common in ANCA-associated vasculitides, diffuse pulmonary hemorrhage, upper or distal respiratory tract findings, nodules or cavitary lesions on thorax CT can be seen as isolated symptoms or signs [2-4]. Lung involvement shows higher mortality and morbidity in patients diagnosed with ANCA-associated vasculitides. An increasing number of publications has reported a relationship between ANCA-associated vasculitides and interstitial lung disease including usual interstitial pneumonia [5, 6]. Chest high-resolution computed tomography (HRCT) findings can be helpful in both diagnosis and predicting prognosis of the disease. However, it is not easy to reach a specific diagnosis based on the imaging findings as the findings of ANCA-associated vasculitides have low specificity. Both airways and lung parenchyma can be involved. In addition to lung involvement, secondary findings such as lymphadenopathy, pleural effusion, pericardial effusion can be detected on chest CT images. The objectives of this review were to describe the main and secondary chest CT findings of ANCA-associated vasculitides and to summarise the differential diagnoses of diseases according to the chest CT findings.

\title{
Granulomatosis with polyangiitis
}

Note:

This manuscript has been peer-reviewed by Prof. Dr. Macit Arıyürek before acceptance.
Hacettepe University Faculty of Medicine, Department of Radiology, Ankara
GPA, previously known as Wegener granulomatosis, is characterized by necrotizing granulomatous inflammation, predominantly in the respiratory system and kidneys. The classic clinical findings consist of upper and lower airway involvement, pulmonary parenchyma involvement, and glomerulonephritis (hematuria, proteinuria, azotemia) [2, 7]. Chest CT demonstrates both parenchyma involvement and airway involvement including the trachea, bronchi and bronchioles. The most common radiological findings of GPA are pulmonary nodules and masses which can be seen in up to 70\% of patients [8]. Lesions are usually bilateral and multiple, and although nodules usually show a random distribution, they have also been described as subpleural in $89 \%$ of patients and peribronchovascular in $41 \%$ [2]. Central cavitation may be seen in up to $50 \%$ of lesions $>2 \mathrm{~cm}$. A surrounding ground glass halo, reverse halo and radiating linear scarring can present as ancillary findings which may help in the diagnosis of GPA. [8, 9] (Figure 1a).

As another feature of GPA which demonstrates the lung parenchyma involvement, ground glass opacity (GGO) and consolidation may occur in up to 50\% of patients [10]. The most common distributions of GGO and consolidation in GPA are peribronchovascular and perihilar. However, random distribution may also be seen. Consolidation may contain air bronchograms and cavitation. Hemorrhage or intraalveolar cellular debris can cause GGO and consolidation $[4,8,11]$ (Figure 1b and 1c).

Following lung parenchyma involvement, the airway is the second most commonly affected area of the thorax in GPA. Both large and small airways can be abnormal. While the abnormality of the large airways is seen in $30 \%$ of patients, wall thickening in the segmental and subsegmental bronchi can occur in approximately $70 \%$ of patients. The subglottic portion is the site most predisposed to involvement. Wall thickening of the trachea is usually circumferential including the posterior membrane of the trachea. It can be smooth or nodular (Figure 2). More often, there is focal involvement, affecting a 2-4 cm area, but segmental or multifocal involvement may also be present. It may eventually lead to subglottic stenosis, which may necessitate tracheostomy [12]. Centrilobular nodules and the tree-in-bud pattern which can result from bronchiolar inflammatory changes may be seen in up to $10 \%$ of patients. In addition to wall thickening and bronchiectasis in distal airways, obstructive atelectasis or pneumonia may occur and be seen on chest CT $[2,8,13]$. 

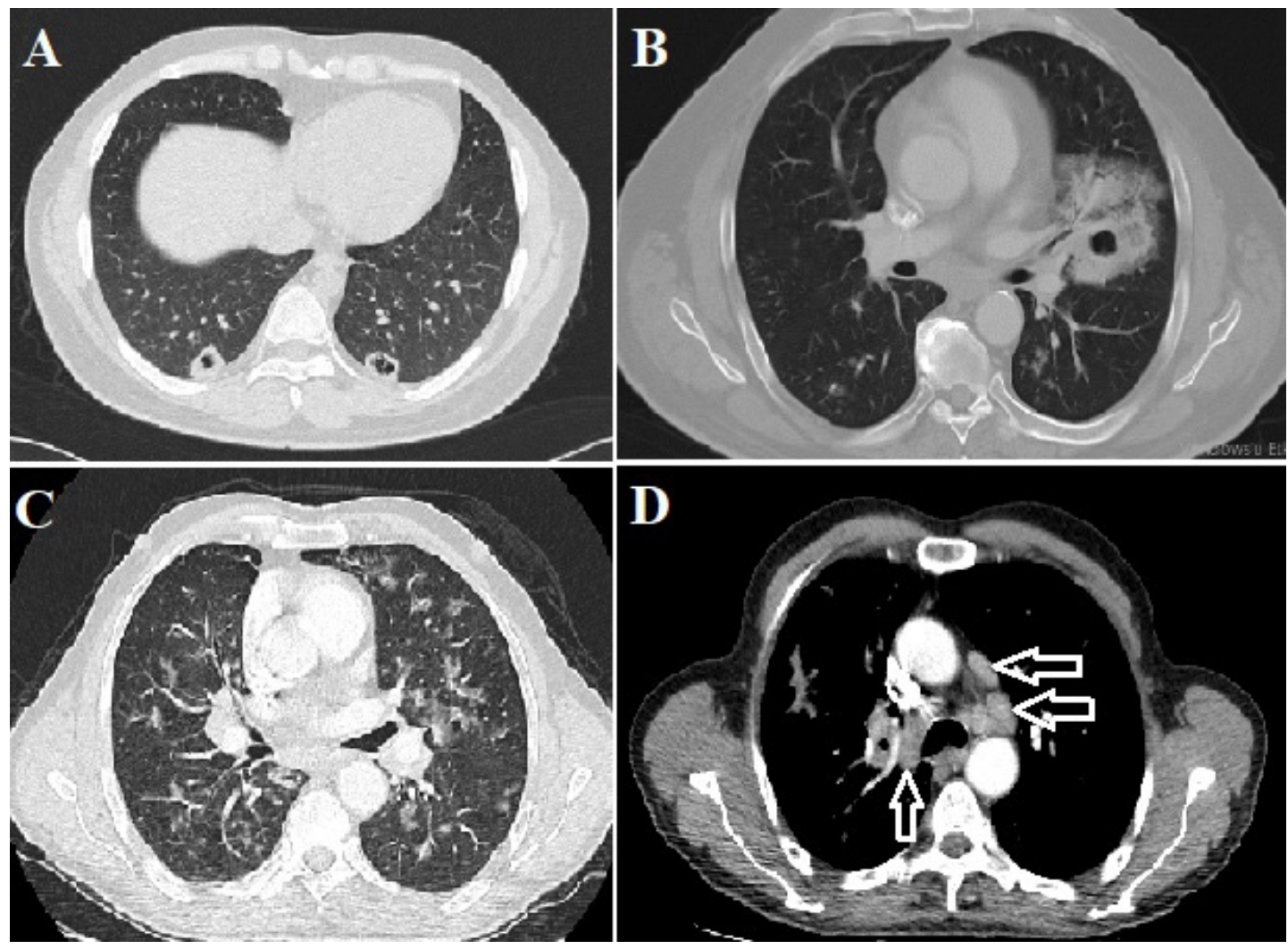

Figure 1: Imaging findings of GPA. Bilateral subpleural nodules which have central cavitation are seen in a 30-year old male patient with GPA (1A). Left hilar consolidation with peripheral GGO seen in a 61-year old male patient. Cavitation within consolidation can also be seen (1B). Axial chest CT image shows bilateral peribronchovascular GGOs in another patient with dyspnea (1C). Enlarged lymph nodes (arrows) are seen in the mediastinal window of the same patient (1D).

Figure 2: Subglottic stenosis of the trachea due to GPA is demonstrated on axial ( $2 \mathrm{~A})$, coronal (2B) and $3 \mathrm{D}$ volume rendering images (2C) (arrows).
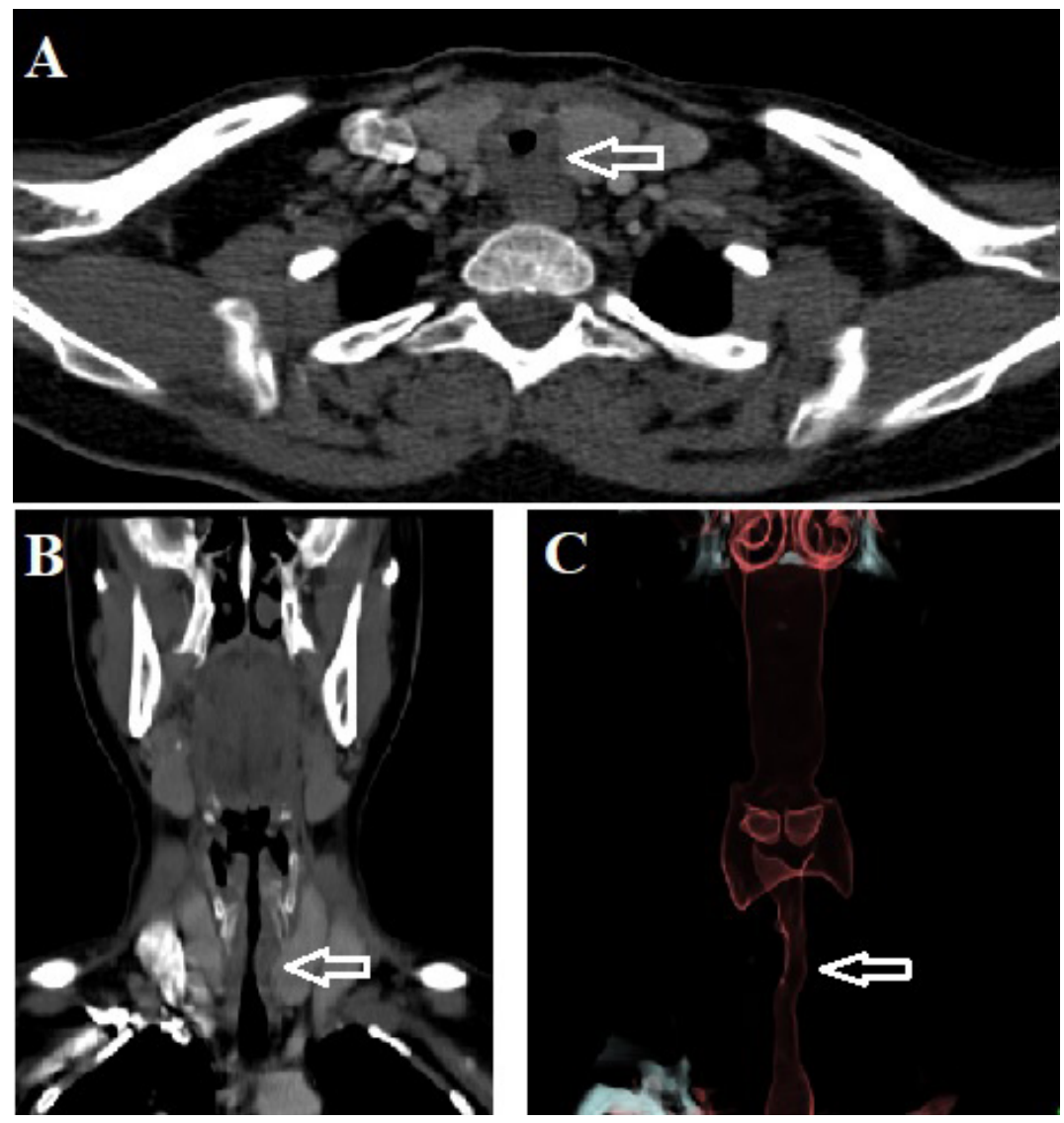
Pleural effusion is the most common pleural abnormality in GPA and may be seen in 12\%-20\% of patients $[8,9]$. In addition to pulmonary abnormalities, although they rarely present in GPA, mediastinal lymphadenopathy, pericardial effusion, myocarditis, valvular dysfunction and heart failure can also be seen (Figure 1d).

\section{Differential diagnosis}

Differential diagnosis of GPA can be applied according to the imaging features on chest CT. While metastasis, sarcoidosis, rheumatoid arthritis and septic emboli can be listed as differential diagnoses for nodules and masses, pneumonia and adenocarcinoma can be listed as differential diagnoses of GGO and consolidation (Figure 3).
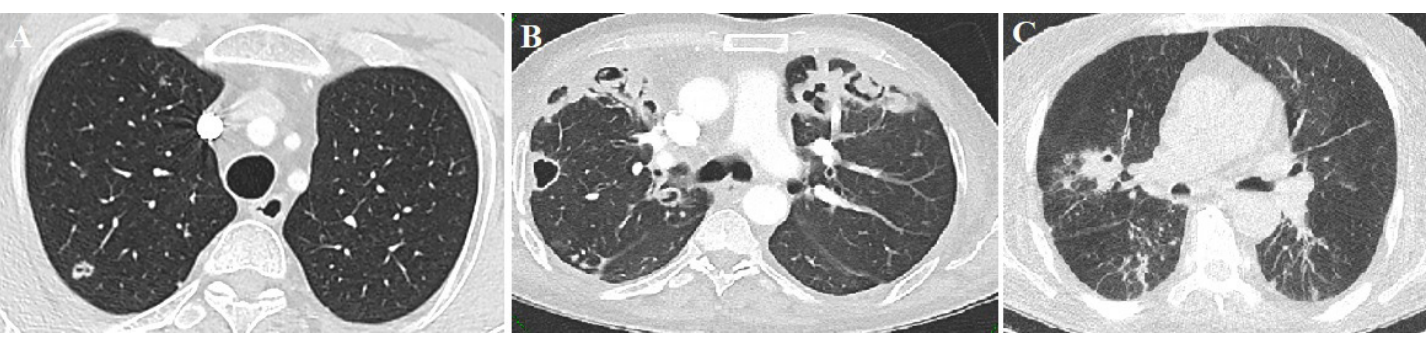

Figure 3: Differential diagnoses of GPA in lung lesions. Cavitary nodules similar to GPA are seen in a patient with colon cancer $(3 \mathrm{~A})$ and another patient with rheumatoid arthritis (3B). Consolidation with cavitation is seen in a patient with Klebsiella pneumonia (3C).

Relapsing polychondritis, tracheobronchopathia osteochondroplastica, and amyloidosis should be considered as differential diagnosis of wall thickening of the trachea. Involvement of the posterior membrane of the trachea can help distinguish GPA from relapsing polychondritis, and tracheobronchopathia osteochondroplastica, both of which spare this area. Moreover, involvement of the subglottic area can also help in the diagnosis of GPA (Figure 4). If the centrilobular nodules and tree-in-bud pattern are seen in GPA, infectious and non-infectious bronchiolitis can be considered in the differential diagnoses.
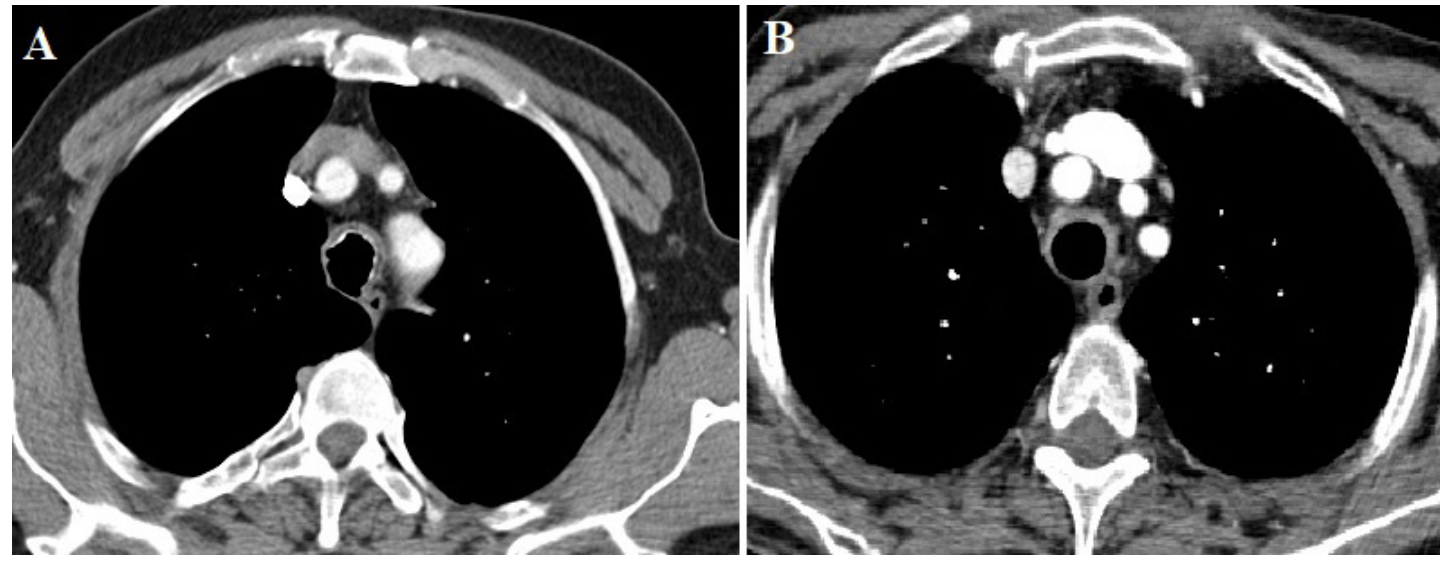

Figure 4: Differential diagnoses of GPA for tracheal lesions. Tracheal thickening seen in a 65-year old male patient with tracheobronchopathia osteochondroplastica (4A). Tracheal thickening in a 50-year old male with relapsing polychondritis is shown on the axial CT image (4B). Sparing of the posterior membrane in these patients can help the differential diagnosis.

\section{Eosinophilic granulomatosis with polyangiitis}

EGPA, also known as Churg-Strauss syndrome, is characterized by a clinical triad of asthma, increased blood eosinophils and necrotizing systemic vasculitis affecting small to medium vessels [14]. The lungs are the most frequently involved organ in EGPA. The main chest CT findings are transient, patchy GGO or consolidation with bilateral and non-segmental distribution, mostly peripheral, without predominance in any lung zone. The pulmonary opacities in EGPA pathologically correspond to eosinophilic pneumonia, extravascular granulomas, and necrotizing vasculitis $[15,16]$. Interlobular septal thickening can also occur due to both the presence of edema and eosinophilic infiltration. Airway involvement is also seen 
as an important chest CT manifestation of EGPA. Bronchial dilatation, wall thickening, centrilobular nodules and mosaic attenuation can be seen due to eosinophilic infiltration of the bronchial wall and asthma on chest CT [15, 17] (Figure 5).
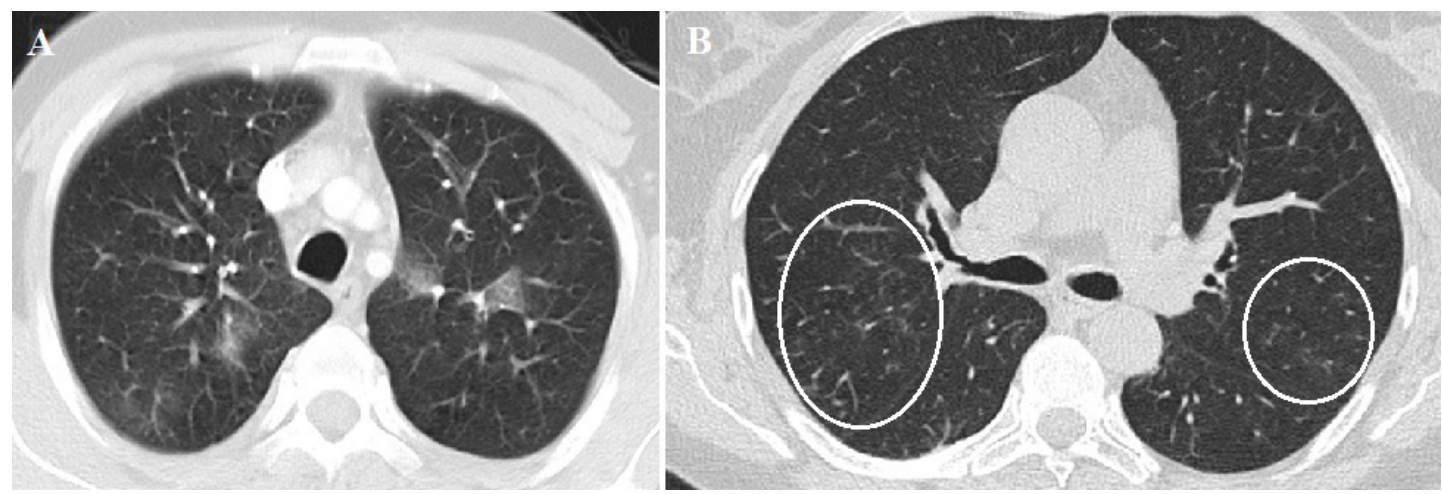

Figure 5: EGPA findings on chest CT. Bilateral patchy GGO in a 28-year old male patient with EGPA (5A). Centrilobular nodules are seen in an another patient with EGPA (5B).

As a secondary finding, pleural effusion can be seen on chest CT in up to 50\% of patients with EGPA. It usually occurs secondary to cardiac involvement $[2,18]$. Cardiac involvement is the leading cause of mortality, and cardiac MRI is more useful for showing cardiac involvement (Figure 6). Interstitial eosinophilic myocardial inflammation, coronary vasculitis and pericardial involvement have been shown to be present at autopsy [19] In a small number of cases, mediastinal lymphadenopathy can also be seen because of reactive hyperplasia and eosinophilic infiltration [20].

\section{Differential diagnosis}

Other eosinophilic lung diseases such as simple pulmonary eosinophilia and chronic eosinophilic pneumonia can be considered first in the differential diagnoses of EGPA. Infectious diseases and pulmonary hemorrhage can also be listed as the differential diagnoses of GGO and consolidations seen in EGPA [2].

Figure 6: MRI findings of a 44-year old male patient with previously diagnosed EGPA. (6A, 6B) Short axis post-contrast phase-sensitive inversion recovery (PSIR) images from the basal and mid left ventricle show inferoseptal subendocardial late gadolinium enhancement (LGE) compatible with fibrosis probably secondary to myocardial ischemia (arrows). (6C, 6D) Short axis cine SSFP images in the end-diastolic (6C) and end-systolic (6D) phases demonstrate a decrease in regional systolic thickening compatible with hypokinesia in corresponding areas (D, arrows).
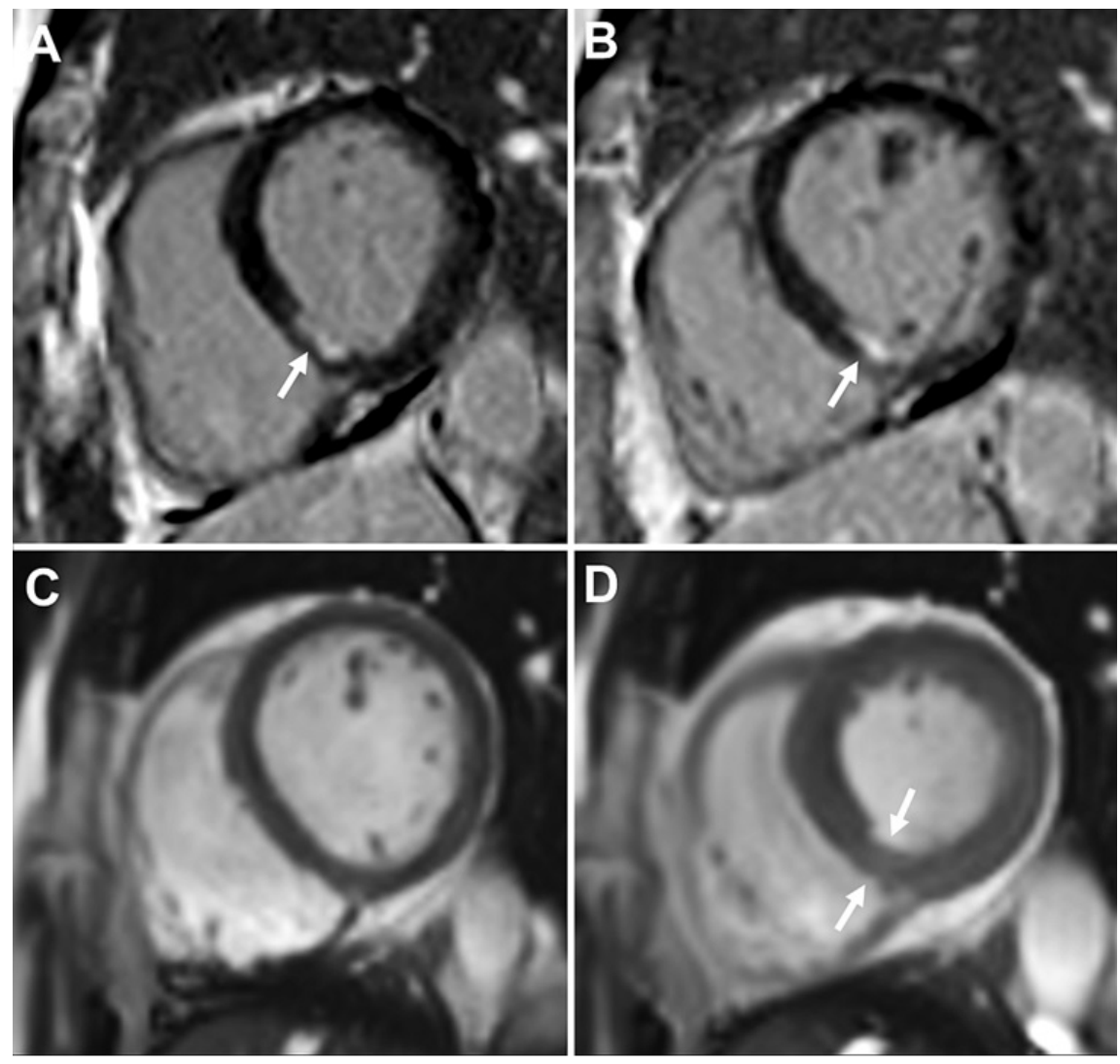
Airway involvement, especially centrilobular nodules, should be differentiated from bronchiolitis such as respiratory bronchiolitis and subacute hypersensitivity bronchiolitis [21] (Figure 7).
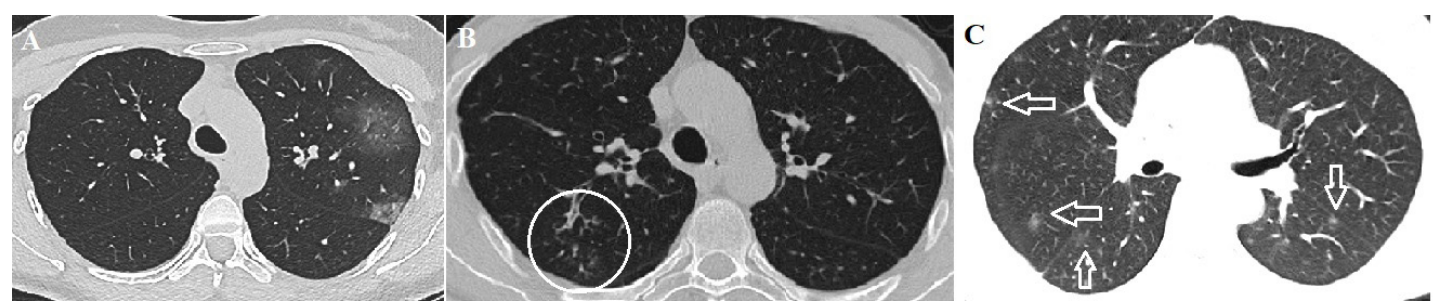

Figure 7: Differential diagnoses of EGPA. GGO seen in a patient with COVID-19 pneumonia (7A) Centrilobular nodules in a patient with haemophilus influenza pneumonia (7B). GGO seen in a 35-year old female with eosinophilic pneumonia $(7 \mathrm{C})$.

\section{Microscopic polyangiitis}

MPA is a systemic non-granulomatous necrotizing vasculitis which predominantly affects small vessels. Although the kidney is the most commonly involved organ observed in $90 \%$ of patients, the lung is involved in a significant percentage (25\%-50\%) of patients $[14,22]$. Lung involvement with interstitial lung disease has been reported to have an adverse impact on the long-term prognosis of MPA [23]. The main imaging findings of MPA are lung opacities secondary to diffuse alveolar hemorrhage (DAH) or hemorrhagic diffuse alveolitis. Although usually bilateral, diffused or patchy GGO with areas of consolidation can be seen on chest CT. While these findings occur in the acute phase of the disease, a reticular pattern and interstitial fibrosis can be seen due to repeated hemorrhage in the chronic phase of MPA. Interstitial lung disease in MPA is associated with poor prognosis. While traction bronchiectasis is seen secondary to interstitial lung disease, bronchial wall thickening and centrilobular opacities are occasionally seen due to airway involvement. Enlargement of mediastinal lymph nodes and pleural effusion have also been reported as additional findings in patients with MPA [23] (Figure 8).
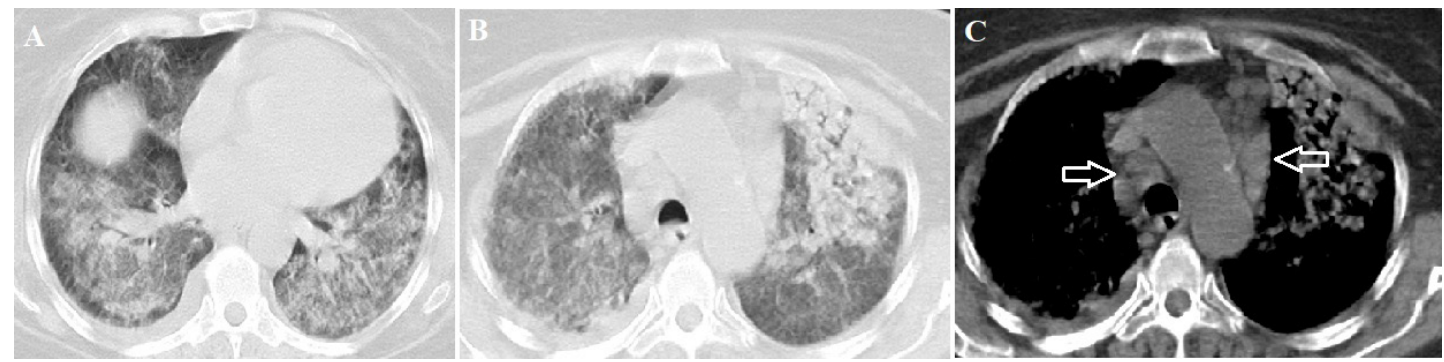

Figure 8: Imaging findings of MPA. Bilateral, diffused GGO with areas of consolidation seen on chest CT (8A and 8B). Enlarged mediastinal lymph nodes are demonstrated in Figure 8C (arrows).

\section{Differential diagnosis}

Differential diagnoses of parenchymal opacities seen in acute phase of MPA, are acute pulmonary edema and infectious diseases. Parenchymal opacities in MPA usually resolve within 1-2 weeks which is slower than in acute pulmonary edema, and this point can help in radiological differential diagnosis (Figure 9).
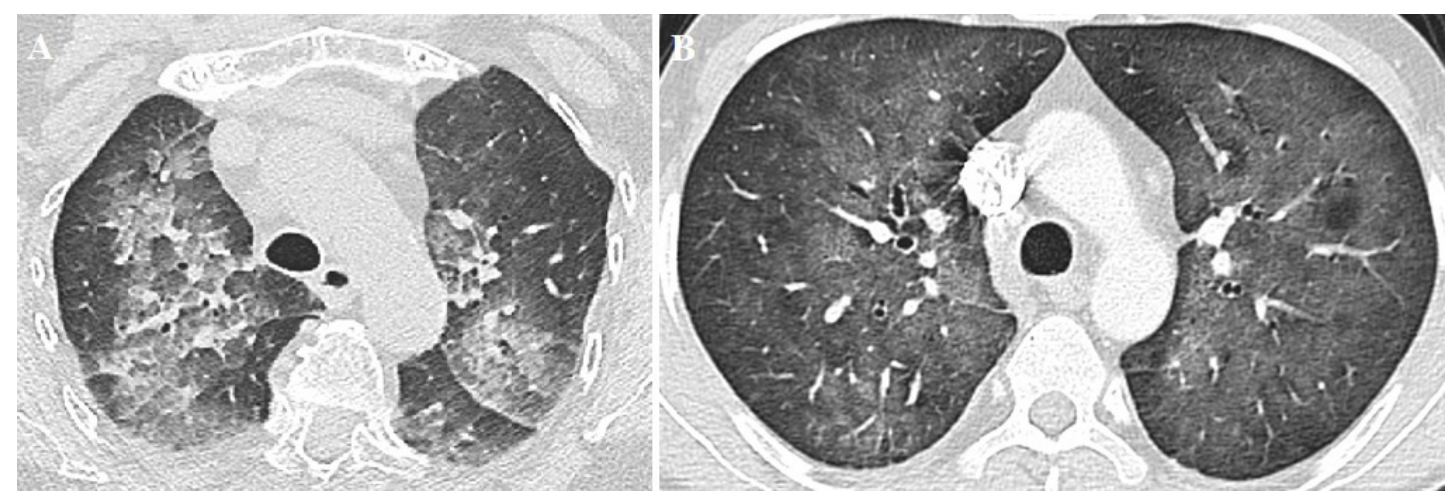

Figure 9: Differential diagnoses of MPA. Bilateral diffuse GGO seen in both pulmonary edema (9A) and pneumocystis jiroveci pneumonia (9B). 
After the acute phase, idiopathic pulmonary fibrosis and pulmonary fibrosis secondary to other diseases can be considered as differential diagnoses of MPA if interstitial lung disease occurs because of recurrent hemorrhage.

ANCA-associated vasculitides describes a group of heterogeneous autoimmune diseases that are different from each other but have some common features. Although imaging findings of ANCA-associated vasculitides are variable and poorly specific, some radiological signs, clinical findings, and laboratory features can be helpful in reaching a specific diagnosis. Both airways and lung parenchyma can be involved. While the most common radiological findings of GPA are pulmonary nodules and masses, the main chest CT findings of EGPA are transient, patchy GGO or consolidation with bilateral and nonsegmental distribution, mostly peripheral, without predominance in any lung zone. Slower resolving bilateral, diffused or patchy GGO with areas of consolidation are suggestive of MPA in the acute phase, whereas a reticular pattern and interstitial fibrosis can be seen due to repeated hemorrhage in the chronic phase of MPA. In addition to lung involvement, secondary findings such as lymphadenopathy, pleural effusion, and pericardial effusion can be detected on chest CT images. Therefore, radiological findings can help the clinician in both diagnosis and prediction of prognosis of ANCA-associated vasculitides.

\section{References}

1. Jennette JC, Falk RJ, Bacon PA, Basu N, Cid MC, Ferrario F, et al. 2012 revised International Chapel Hill Consensus Conference Nomenclature of Vasculitides. Arthritis Rheum. 2013;65(1):1-11.

2. Chung MP, Yi CA, Lee HY, Han J, Lee KS. Imaging of pulmonary vasculitis. Radiology. 2010;255(2):322-41.

3. Brown KK. Pulmonary vasculitis. Proc Am Thorac Soc. 2006;3(1):48-57.

4. Hansell DM. Small-vessel diseases of the lung: CT-pathologic correlates. Radiology. 2002;225(3):639-53.

5. Suzuki A, Sakamoto S, Kurosaki A, Kurihara Y, Satoh K, Usui Y, et al. Chest High-Resolution CT Findings of Microscopic Polyangiitis: A Japanese First Nationwide Prospective Cohort Study. AJR Am J Roentgenol. 2019:1-11.

6. Alba MA, Flores-Suarez LF, Henderson AG, Xiao H, Hu P, Nachman PH, et al. Interstital lung disease in ANCA vasculitis. Autoimmun Rev. 2017;16(7):722-9.

7. Frankel SK, Cosgrove GP, Fischer A, Meehan RT, Brown KK. Update in the diagnosis and management of pulmonary vasculitis. Chest. 2006;129(2):452-65.

8. Martinez F, Chung JH, Digumarthy SR, Kanne JP, Abbott GF, Shepard JA, et al. Common and uncommon manifestations of Wegener granulomatosis at chest CT: radiologic-pathologic correlation. Radiographics. 2012;32(1):51-69.

9. Cordier JF, Valeyre D, Guillevin L, Loire R, Brechot JM. Pulmonary Wegener's granulomatosis. A clinical and imaging study of 77 cases. Chest. 1990;97(4):906-12.

10. Sheehan RE, Flint JD, Muller NL. Computed tomography features of the thoracic manifestations of Wegener granulomatosis. J Thorac Imaging. 2003;18(1):34-41.

11. Lenclud C, De Vuyst P, Dupont E, Depierreux M, Ketelbant P, Goldman M. Wegener's granulomatosis presenting as acute respiratory failure with anti-neutrophil-cytoplasm antibodies. Chest. 1989;96(2):345-7.

12. Aberle DR, Gamsu G, Lynch D. Thoracic manifestations of Wegener granulomatosis: diagnosis and course. Radiology. 1990;174(3 Pt 1):703-9.

13. Lee KS, Kim TS, Fujimoto K, Moriya H, Watanabe H, Tateishi U, et al. Thoracic manifestation of Wegener's granulomatosis: CT findings in 30 patients. Eur Radiol. 2003;13(1):43-51.

14. Jennette JC, Falk RJ. Small-vessel vasculitis. N Engl J Med. 1997;337(21):1512-23.

15. Silva CI, Muller NL, Fujimoto K, Johkoh T, Ajzen SA, Churg A. Churg-Strauss syndrome: high resolution CT and pathologic findings. J Thorac Imaging. 2005;20(2):74-80.

16. Katzenstein AL. Diagnostic features and differential diagnosis of Churg-Strauss syndrome in the lung. A review. Am J Clin Pathol. 2000;114(5):767-72.

17. Feragalli B, Mantini C, Sperandeo M, Galluzzo M, Belcaro G, Tartaro A, et al. The lung in systemic vasculitis: radiological patterns and differential diagnosis. Br J Radiol. 2016;89(1061):20150992.

18. Johkoh T, Muller NL, Akira M, Ichikado K, Suga M, Ando M, et al. Eosinophilic lung diseases: diagnostic accuracy of thinsection CT in 111 patients. Radiology. 2000;216(3):773-80.

19. Worthy SA, Muller NL, Hansell DM, Flower CD. Churg-Strauss syndrome: the spectrum of pulmonary CT findings in 17 patients. AJR Am J Roentgenol. 1998;170(2):297-300.

20. Lesens O, Hansmann Y, Nerson J, Pasquali J, Gasser B, Wihlm J, et al. Severe Churg-Strauss syndrome with mediastinal lymphadenopathy treated with interferon therapy. Eur J Intern Med. 2002;13(7):458.

21. Okada F, Ando Y, Yoshitake S, Ono A, Tanoue S, Matsumoto S, et al. Clinical/pathologic correlations in 553 patients with primary centrilobular findings on high-resolution CT scan of the thorax. Chest. 2007;132(6):1939-48.

22. Guillevin L, Durand-Gasselin B, Cevallos R, Gayraud M, Lhote F, Callard P, et al. Microscopic polyangiitis: clinical and laboratory findings in eighty-five patients. Arthritis Rheum. 1999;42(3):421-30.

23. Suzuki M, Okata H, Sakata H, Sato H. Microscopic polyangiitis masquerading as a pancreatic neoplasm with multiple lung metastases. BMJ Case Rep. 2019;12(8). 